\title{
Exact solutions of a Flat Full Causal Bulk viscous FRW cosmological model through factorization
}

\author{
O. Cornejo-Pérez \\ Facultad de Ingeniería, Universidad Autónoma de Querétaro, \\ Centro Universitario Cerro de las Campanas, 76010 Santiago de Querétaro, Mexico \\ J. A. Belinchón \\ Departamento de Física Atómica, Molecular y Nuclear. Universidad Complutense de Madrid, E-28040 Madrid, España
}

(Date text: June 26, 2018)

\begin{abstract}
We study the classical flat full causal bulk viscous FRW cosmological model through the factorization method. The method shows that there exists a relationship between the viscosity parameter $s$ and the parameter $\gamma$ entering the equations of state of the model. Also, the factorization method allows to find some new exact parametric solutions for different values of the viscous parameter $s$. Special attention is given to the well known case $s=1 / 2$, for which the cosmological model admits scaling symmetries. Furthermore, some exact parametric solutions for $s=1 / 2$ are obtained through the Lie group method.
\end{abstract}

Keywords: Exact solutions, Full Causal Bulk viscosity, factorization method, Lie groups.

\section{INTRODUCTION.}

Factorization of linear second order differential equations is a well established method to find exact solutions through algebraic procedures. It was widely used in quantum mechanics and developed since Schrodinger's works on the factorization of the Sturm-Liouville equation. At the present time, very good informative reviews on the factorization method can be found in open literature (see for instance [1, 2] ). However, in recent times the factorization method has been applied to find exact solutions of nonlinear ordinary differential equations (ODE) [3 $[7]$. In [4], based on previous Berkovich's works [3], it has been provided a systematic way to apply the factorization method to nonlinear second order ODE. In [5], Wang and Li extended the application to more complex nonlinear second and third order ODE. The factorization of some ODE may be restricted due to constraints which appear in a natural way within the factorization procedure. However, here it is shown that by performing transformation of coordinates, one can be able to get exact parametric solutions of an ODE which does not allow its factorization or presents cumbersome constraints.

The purpose of the present work is to apply the factorization method to study the full causal bulk viscous cosmological model with flat FRW symmetries. Since the Misner [8] suggestion stressing the fact that the observed large scale isotropy of the Universe may be due to the action of the neutrino viscosity when the Universe was about one second old, there have been numerous works pointing out the importance of the physical processes involving viscous effects in the evolution of the Universe (see for instance [9]). Due to such assumption, dissipative processes are supposed to play a fundamental role in the evolution of the early Universe.

The theory of relativistic dissipative fluids, created by Eckart [10] and Landau and Lifshitz [11] has many drawbacks, and it is known that it is incorrect in several respects mainly those concerning causality and stability. Israel [12] formulates a new theory in order to solve these drawbacks. This theory was latter developed by Israel and Stewart [13] into what is called transient or extended irreversible thermodynamics. The best currently available theory for analyzing dissipative processes in the Universe is the full causal thermodynamics developed by Israel and Stewart [13], Hiscock and Lindblom [14] and Hiscock and Salmonson [15]. The full causal bulk viscous thermodynamics has been extensively used to study the evolution of the early Universe and some astrophysical process [16, 17].

The paper is organized as follows. In Section II, we start by reviewing the main components of a flat bulk viscous FRW cosmological model, and introduce the factorization technique as applied to the cosmological model. Field equations (FE) of the classical bulk viscous FRW cosmological model [17] reduce to a single nonlinear second order ODE, the fundamental dynamical equation for the Hubble rate. By performing a transformation of both the dependent and independent variables and using the factorization method, this equation is transformed into a nonlinear first order ODE. The order reduction of the equation for the Hubble rate allows to find a variety of new exact parametric solutions of the FE for the viscous FRW cosmological model. Furthermore, the factorization technique provides relationships for parameters entering the factorized equation. Then, a noteworthy result is that the viscosity parameter $s$ is not longer assumed to be independent of the values of parameter $\gamma$. Such parameter relationships have not been previously reported. In Section III, several particular models for $s \neq 1 / 2$ are studied. We obtain new exact parametric solutions through factorization and compare with the ones obtained by several authors [18 25] who use different approaches. Section IV is devoted to the special case $s=1 / 2$, for which the model admits scaling symmetries. The scaling solution, 
previously studied by many authors is obtained. In order to obtain more new solutions and compare the solutions obtained through factorization for $s=1 / 2$, we consider the Lie group method for this special case in Section V. Some conclusions end up the paper in Section VI.

\section{THE MODEL.}

We consider a flat FRW Universe with line element

$$
d s^{2}=-d t^{2}+f^{2}(t)\left(d x^{2}+d y^{2}+d z^{2}\right),
$$

where the energy-momentum tensor of a bulk viscous cosmological fluid is given by [17]:

$$
T_{i}^{k}=(\rho+p+\Pi) u_{i} u^{k}+(p+\Pi) \delta_{i}^{k},
$$

where $\rho$ is the energy density, $p$ the thermodynamic pressure, $\Pi$ the bulk viscous pressure and $u_{i}$ the four-velocity satisfying the condition $u_{i} u^{i}=-1$. We use the units $8 \pi G=c=1$. The gravitational field equations together with the continuity equation, $T_{i ; k}^{k}=0$, are given as follows

$$
\begin{aligned}
2 \dot{H}+3 H^{2} & =-p-\Pi, \\
3 H^{2} & =\rho, \\
\Pi+\tau \dot{\Pi} & =-3 \xi H-\frac{1}{2} \tau \Pi\left(3 H+\frac{\dot{\tau}}{\tau}-\frac{\dot{\xi}}{\xi}-\frac{\dot{T}}{T}\right), \\
\dot{\rho} & =-3(\gamma \rho+\Pi) H,
\end{aligned}
$$

where $H=\dot{f} / f$. In order to close the system of equations we are assuming the following equations of state [17]

$$
p=(\gamma-1) \rho, \quad \xi=\alpha \rho^{s}, \quad T=\beta \rho^{r}, \quad \tau=\xi \rho^{-1}=\alpha \rho^{s-1},
$$

where $T$ is the temperature, $\xi$ the bulk viscosity coefficient and $\tau$ the relaxation time. The parameters satisfy $\gamma \in[1,2]$, $s \geq 0$, and $r=\left(1-\frac{1}{\gamma}\right)$. The growth of entropy has the following behavior

$$
\Sigma(t) \approx-3 k_{B}^{-1} \int_{t_{0}}^{t} \Pi H f^{3} T^{-1} d t .
$$

The Israel-Stewart-Hiscock theory is derived under the assumption that the thermodynamical state of the fluid is close to equilibrium, i.e., the non-equilibrium bulk viscous pressure should be small when compared to the local equilibrium pressure $|\Pi|<<p=(\gamma-1) \rho$. Then, we may define the $l(t)$ parameter as: $l=|\Pi| / p$. If this condition is violated then one is effectively assuming that the linear theory also holds in the nonlinear regime far from equilibrium. For a fluid description of the matter, the condition ought to be satisfied.

To see if a cosmological model inflates or not it is convenient to introduce the deceleration parameter $q=d H^{-1} / d t-$ 1. The positive sign of the deceleration parameter corresponds to standard decelerating models, whereas the negative sign indicates inflation.

The fundamental dynamical equation for the Hubble rate is given by [17]

$$
\ddot{H}-A \frac{\dot{H}^{2}}{H}+\left(3 H+C H^{2-2 s}\right) \dot{H}+D H^{3}+E H^{4-2 s}=0,
$$

where

$$
A=(1+r)=2-\frac{1}{\gamma}, \quad B=3, \quad C=3^{1-s}, \quad D=\frac{9}{4}(\gamma-2), \quad E=\frac{1}{2} 3^{2-s} \gamma
$$

Let us perform the following transformation of the dependent and independent variables

$$
H=y^{1 / 2}, \quad d \eta=y^{1 / 2} d t
$$

then Eq. (9) turns into

$$
\frac{d^{2} y}{d \eta^{2}}-\frac{A}{2 y}\left(\frac{d y}{d \eta}\right)^{2}+\left(3+C y^{\frac{1}{2}-s}\right) \frac{d y}{d \eta}+2 y\left(D+E y^{\frac{1}{2}-s}\right)=0
$$


Let us consider now the following factorization scheme [4, 5]. The nonlinear second order equation

$$
y^{\prime \prime}+f(y) y^{\prime 2}+g(y) y^{\prime}+h(y)=0
$$

where $y^{\prime}=\frac{d y}{d \eta}=D_{\eta} y$, can be factorized in the form

$$
\left[D_{\eta}-\phi_{1}(y) y^{\prime}-\phi_{2}(y)\right]\left[D_{\eta}-\phi_{3}(y)\right] y=0
$$

under the conditions

$$
\begin{aligned}
& f(y)=-\phi_{1}, \\
& g(y)=\phi_{1} \phi_{3} y-\phi_{2}-\phi_{3}-\frac{d \phi_{3}}{d y} y, \\
& h(y)=\phi_{2} \phi_{3} y .
\end{aligned}
$$

If we assume $\left[D_{\eta}-\phi_{3}(y)\right] y=\Omega(y)$, then the factorized Eq. (14) can be rewritten as

$$
\begin{aligned}
y^{\prime}-\phi_{3} y & =\Omega, \\
\Omega^{\prime}-\left(\phi_{1} y^{\prime}+\phi_{2}\right) \Omega & =0 .
\end{aligned}
$$

We can introduce the functions $\phi_{i}$ by comparing Eqs. (12) and (13). Then, $\phi_{1}=\frac{A}{2 y}, \phi_{2}=a_{1}^{-1}$ and $\phi_{3}=$ $2 a_{1}\left(D+E y^{\frac{1}{2}-q}\right)$, where $a_{1}(\neq 0)$ is an arbitrary constant, are proposed.

Eq. (19) can be easily solved for the chosen factorizing functions obtaining as result $\Omega=\kappa_{1} e^{\eta / a_{1}} y^{A / 2}$, where $\kappa_{1}$ is an integration constant. Then, Eq. (18) turns into the equation

$$
y^{\prime}-2 a_{1}\left(D+E y^{\frac{1}{2}-s}\right) y-\kappa_{1} e^{\eta / a_{1}} y^{A / 2}=0
$$

whose solution is also solution of Eq. (12).

Furthermore, the following relationship is obtained from Eq. (16),

$$
A a_{1} D-a_{1}^{-1}-2 a_{1} D+a_{1} E(A-3+2 s) y^{\frac{1}{2}-s}=3+C y^{\frac{1}{2}-s} .
$$

Eq. (21) is a noteworthy result which provides the explicit form of $a_{1}$ and the relationship among the parameters entering Eq. (12). Then, the viscous parameter $s$ as a function of parameter $\gamma$ is obtained. By comparing both sides of Eq. (21) and assuming $r=1-\frac{1}{\gamma}$, leads to obtain:

$$
s(\gamma)_{ \pm}=\frac{ \pm \sqrt{2}+\gamma^{3 / 2}}{2 \gamma^{3 / 2}}
$$

Then, $s_{-} \in[0, .25] \forall \gamma \in[1.2599,2]$, and $s_{+} \in(.75,1.2071068] \forall \gamma \in[1,2)$. Also, the explicit form of $a_{1}$ is

$$
a(\gamma)_{1 \pm}= \pm \frac{2 \gamma^{1 / 2}}{3\left(\sqrt{2} \mp \gamma^{1 / 2}\right)}
$$

Then, $a_{1-} \in[-1 / 3,-.29499] \forall \gamma \in[1.2599,2]$, and $a_{1+} \in[1.60947, \infty) \forall \gamma \in[1,2)$.

We find the following significative values

\begin{tabular}{|c|c|c|c|c|}
\hline$\gamma$ & $s_{-}$ & $a_{1-}$ & $s_{+}$ & $a_{1+}$ \\
\hline \hline 1 & & & 1.2071 & 1.6095 \\
\hline$\frac{4}{3}$ & $4.0721 \times 10^{-2}$ & -0.29966 & 0.95928 & 2.9663 \\
\hline 2 & $\frac{1}{4}$ & $-\frac{1}{3}$ & & \\
\hline
\end{tabular}

The main difference of these results from other approaches is expressed through Eq. (22), which represents an advantage of the factorization method as opposed to different approaches studied by other authors. This equation provides the relationship between the parameters $s$ and $\gamma$ in such a way that by fixing $s$ we get a particular value of $\gamma$. 
The main dynamical variables of the FE are given in parametric form as follows

$$
\begin{aligned}
f(\eta) & =f_{0} \exp \left(\eta-\eta_{0}\right) \\
H(\eta) & =y^{1 / 2}(\eta) \\
q(\eta) & =y^{1 / 2}(\eta) \frac{d}{d \eta}\left(\frac{1}{H(\eta)}\right)-1, \\
\rho(\eta) & =3 y(\eta) \\
p(\eta) & =3(\gamma-1) y(\eta) \\
\Pi(\eta) & =-\left(3 \gamma y(\eta)+\frac{d y}{d \eta}\right), \\
l(\eta) & =\frac{|\Pi|}{p}, \\
\Sigma(\eta) & =-3 k_{B} \int \Pi(\eta) f^{3}(\eta) H(\eta) T(\eta)^{-1} y(\eta)^{-1 / 2} d \eta
\end{aligned}
$$

The authors have not been able to find the most general solution of Eq. (20). However, this equation can be studied for some specific cases providing particular solutions of physical interest. In Sections III and IV, the cosmological solutions as obtained for the viscosity parameter $s \neq 1 / 2$ and $s=1 / 2$ are studied.

\section{SOLUTION WITH $s \neq 1 / 2$.}

In this section, some particular cases of Eq. (20) for $s \neq 1 / 2$ are studied to obtain exact particular solutions of FE (3)-(6). By setting $\kappa_{1}=0$, Eq. (20) simplifies as

$$
y^{\prime}-2 a_{1}\left(D+E y^{\frac{1}{2}-s}\right) y=0
$$

whose solution is given by

$$
y(\eta)=\left(\kappa_{2} e^{a_{1} D(2 s-1) \eta}-\frac{E}{D}\right)^{2 /(2 s-1)},
$$

where $\kappa_{2}$ is an integration constant. Therefore, the parametric form of the time function is obtained from Eq. (11) as follows

$$
t(\eta)=\int y^{-1 / 2}(\eta) d \eta=\int\left(\kappa_{2} e^{a_{1} D(2 s-1) \eta}-\frac{E}{D}\right)^{1 /(1-2 s)} d \eta
$$

\section{A. Case $s=0$.}

The first special case considered corresponds to $s=0$, which means that the bulk viscosity coefficient $\xi=$ const. The following particular solution is obtained

$$
y(\eta)=\left(\kappa_{2} e^{-a_{1} D \eta}-\frac{E}{D}\right)^{-2}, \quad \text { and } \quad t(\eta)=-\frac{1}{D a_{1}}\left(\kappa_{2} e^{-\eta D a_{1}}+\eta E a_{1}\right)
$$

Eqs. (22) and (23) provide the corresponding constant parameters $a_{1-}=-0.295$ and $\gamma=\sqrt[3]{2}$, respectively. A particular equation of state is obtained once again through Eq. (22). In Figs. 1 and 2, the behavior of the FE main quantities for different values of constant $\kappa_{2}$ is plotted.

As we can see, the solution for $\kappa_{2}=-1$ is non-singular since $\rho(0)=$ const. For $\kappa_{2}=-2$ and $\kappa_{2}=-3$, the energy density has a singular behavior when $t=0$, since it runs to infinity when time tends to zero, i.e., $\rho(0) \rightarrow \infty$. The bulk viscosity, $\Pi$, is negative for all values of $t$, i.e., $\Pi(t)<0 \forall t \in \mathbb{R}^{+}$, which is a thermodynamically consistent result as expected for $\kappa_{2}=-1$. For $\kappa_{2}=-2$ and $\kappa_{2}=-3$, the solution is valid only when $t>t_{0}$, i.e., $\Pi(t)<0 \forall t>t_{0}$, while $\Pi(t \rightarrow 0)>0$. Then, for this interval of time, $t \in\left(0, t_{0}\right)$, the solution has no physical meaning. The entropy behaves like a strictly growing time function; then, there are a large amount of comoving entropy during the expansion of the 

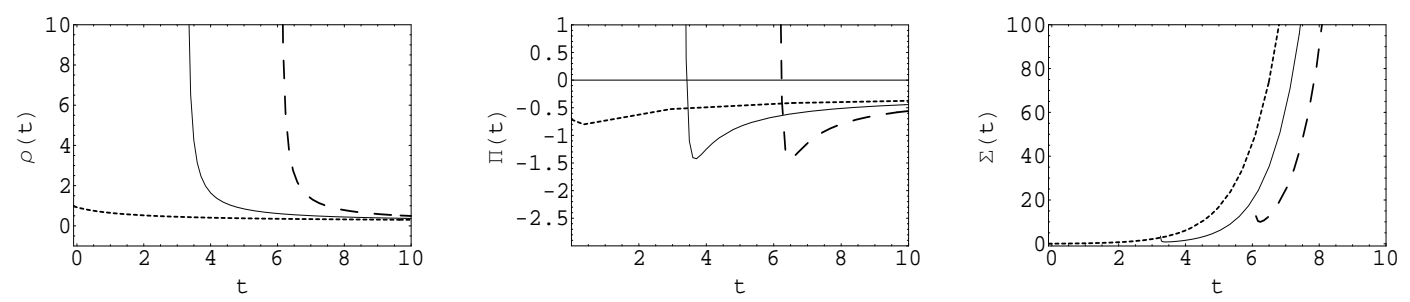

FIG. 1: Solution with $s=0$. Plots of energy density $\rho(t)$, bulk viscosity $\Pi(t)$ and entropy $\Sigma(t)$. Dashed line for $\kappa_{2}=-1$. Solid line for $\kappa_{2}=-2$. Long dashed line for $\kappa_{2}=-3$.
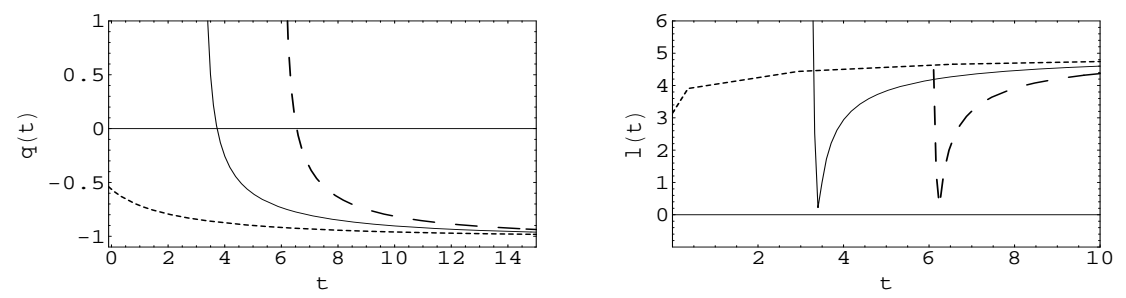

FIG. 2: Solution with $s=0$. Plots of the deceleration parameter $q(t)$ and parameter $l(t)$. Plots of energy density $\rho(t)$, bulk viscosity $\Pi(t)$ and entropy $\Sigma(t)$. Dashed line for $\kappa_{2}=-1$. Solid line for $\kappa_{2}=-2$. Long dashed line for $\kappa_{2}=-3$.

universe. The deceleration parameter runs from $q(0)=-0.5$ to $q(t)=-1$. Then, the solution is accelerating, i.e., it is inflationary. The deceleration parameter tends to -1 as $t \rightarrow \infty$ (accelerating solutions) but shows a singular behavior when time runs to zero. The parameter $l(t)$ shows that all the plotted solutions are far from equilibrium since they are inflationary solutions, which is a consistent result. To the best of our knowledge this solution is new.

\section{B. Case $s=1 / 4$.}

The second case considered corresponds to $s=1 / 4$. In this case, Eqs. (22) and (23) provide $a_{1}=-1 / 3$ and $\gamma=2$. Therefore, Eq. (201) simplifies as

$$
y^{\prime}+2(3)^{3 / 4} y^{5 / 4}-\kappa_{1} e^{-3 \eta} y^{3 / 4}=0 .
$$

If we perform the transformation $z=y^{1 / 4}$ in Eq. (36), then we get the Riccati equation

$$
z^{\prime}+\frac{3^{3 / 4}}{2} z^{2}-\frac{1}{4} \kappa_{1} e^{-3 \eta}=0
$$

whose general solution is given in terms of Bessel $J_{n}$ and Neumman $N_{n}$ functions,

$$
z(\eta)=-\xi(\eta) \frac{J_{1}(\xi(\eta))+\kappa_{2} N_{1}(\xi(\eta))}{J_{0}(\xi(\eta))+\kappa_{2} N_{0}(\xi(\eta))}
$$

where $\xi(\eta)=\frac{\sqrt{2 \kappa_{1}}}{2 \cdot 3^{3 / 8}} e^{-3 \eta / 2}$ and $\kappa_{2}$ is an integration constant. Therefore, the following special solution for Eq. (36) is obtained:

$$
y(\eta)=\left(\xi(\eta) \frac{J_{1}(\xi(\eta))+\kappa_{2} N_{1}(\xi(\eta))}{J_{0}(\xi(\eta))+\kappa_{2} N_{0}(\xi(\eta))}\right)^{4}, \quad t(\eta)=\int^{\eta}\left(\xi(\eta) \frac{J_{1}(\xi(\eta))+\kappa_{2} N_{1}(\xi(\eta))}{J_{0}(\xi(\eta))+\kappa_{2} N_{0}(\xi(\eta))}\right)^{-2} d \eta .
$$

In order to study the behavior of the FE dynamical variables in their parametric form, the calculation of Eq. (39) has been numerically addressed. The solution depends strongly on the value of the numerical constants, in such a way that our solution is physical only for $\kappa_{2}<0$ and for negative and relatively small values $(<20)$ of $\kappa_{1}$. Numerical analysis of the solution plotted in Fig. 3 shows that the solution is singular since the energy density tends to infinity 
when $t \rightarrow 0$. The bulk viscosity is positive, $\Pi>0$, in the region $\left(0, t_{*}\right)$ so the solution has physical meaning only when $t>t_{*}$, for this era $\Pi$ becomes negative as expected from the thermodynamical point of view and tending to zero in the large time limit. In the same interval of time $\left(0, t_{*}\right)$ the entropy production is negative, $\Sigma(t)<0$ (unphysical situation), nevertheless when $t>t_{*}$, a large amount of comoving entropy is produced during the expansion of the universe.
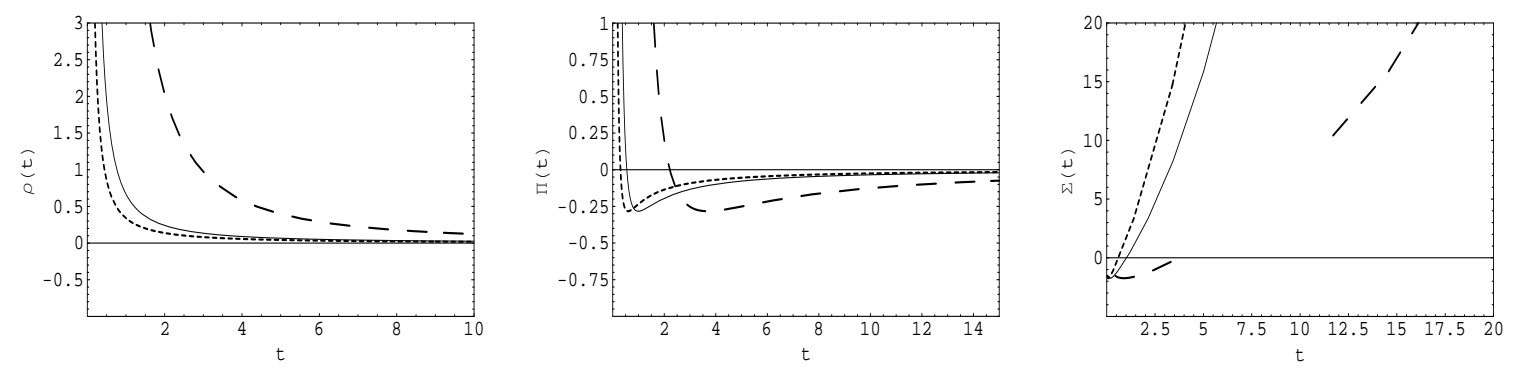

FIG. 3: Solution with $s=1 / 4$ and $\gamma=2$. Plots of energy density $\rho(t)$, bulk viscosity $\Pi(t)$ and entropy $\Sigma(t)$. Dashed line for $\kappa_{1}=4, \kappa_{2}=-10$. Solid line for $\kappa_{1}=19, \kappa_{2}=-3$. Long dashed line for $\kappa_{1}=3, \kappa_{2}=-0.7$.

Regarding the dynamical behavior of solution (39), in Fig. 4 the behavior of parameters $q$ and $l$ has been plotted. As we can see, the deceleration parameter shows that the universe starts in a non-inflationary phase, but quickly entering a inflationary one since $q<0$. The plots of $l(t)$ are consistent with this behavior, showing that the solution starts in a thermodynamical equilibrium but in a finite time they are far from equilibrium since they are inflationary solutions.
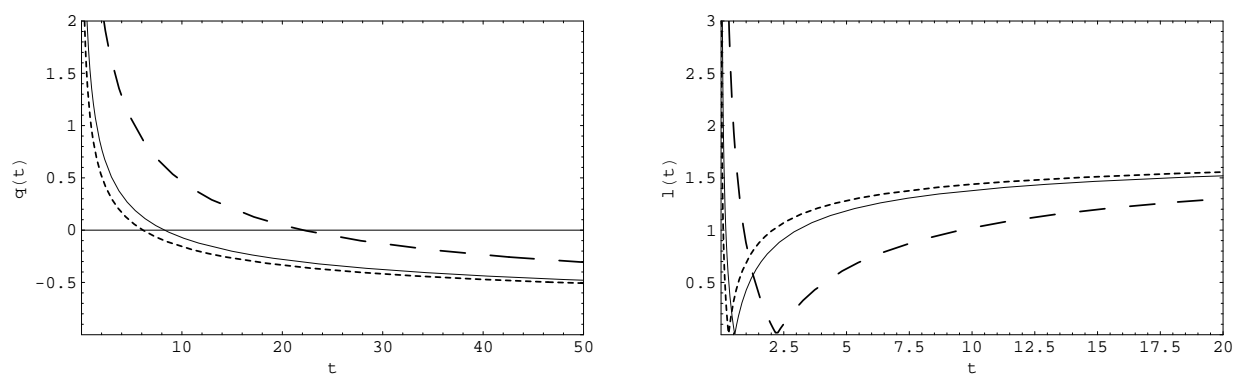

FIG. 4: Solution with $s=1 / 4$ and $\gamma=2$. Plots of the deceleration parameter $q(t)$ and parameter $l(t)$. Dashed line for $\kappa_{1}=4$, $\kappa_{2}=-10$. Solid line for $\kappa_{1}=19, \kappa_{2}=-3$. Long dashed line for $\kappa_{1}=3, \kappa_{2}=-0.7$.

A similar solution has been obtained by Mak et al [25] but, as we have shown, our solution is qualitatively different, with a very different physical meaning.

\section{A particular solution for the case $s=1 / 4$.}

If we set $\kappa_{1}=0$ in Eq. (36), then we get the very simple ODE

$$
y^{\prime}+2(3)^{3 / 4} y^{5 / 4}=0,
$$

whose solution is given as

$$
y(\eta)=\left(\frac{(3)^{3 / 4}}{2} \eta+\kappa_{2}\right)^{-4}, \quad \text { and } \quad t(\eta)=\frac{1}{4} \sqrt{3} \eta^{3}+\frac{1}{2} 3^{\frac{3}{4}} \eta^{2} \kappa_{2}+\eta \kappa_{2}^{2},
$$

where $\kappa_{2}$ is an integration constant. In Figs. 5 and 6 the behavior of the FE main quantities has been plotted for different values of the constant $\kappa_{2}$. 

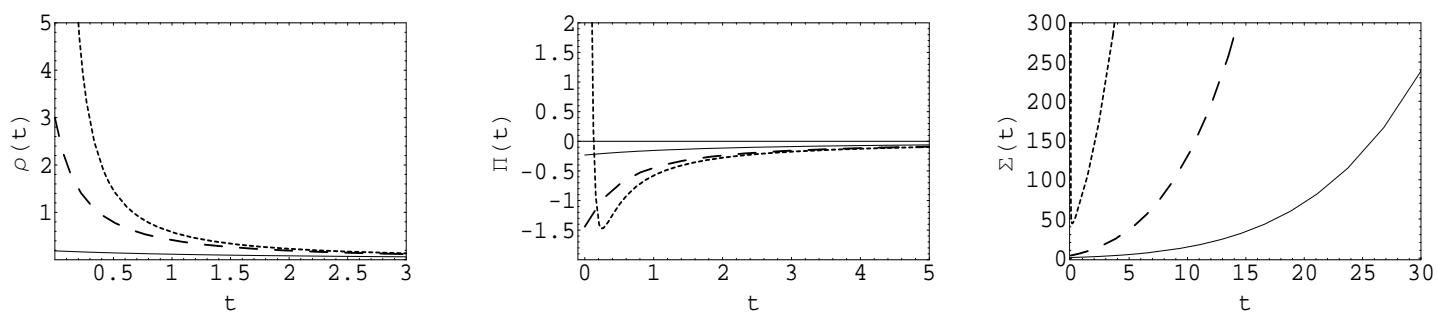

FIG. 5: Particular solution for $s=1 / 4$. Plots of energy density $\rho(t)$, bulk viscosity $\Pi(t)$ and entropy $\Sigma(t)$. Dashed line for $\kappa_{2}=0$. Long dashed line for $\kappa_{2}=1$. Solid line for $\kappa_{2}=2$.
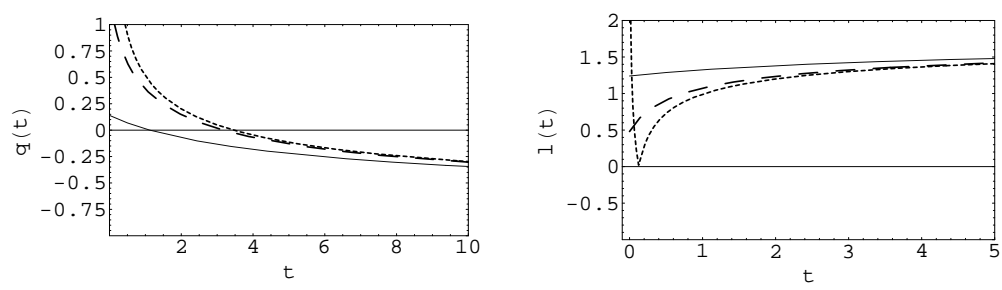

FIG. 6: Particular solution for $s=1 / 4$. Plots of the deceleration parameter $q(t)$ and parameter $l(t)$. Dashed line for $\kappa_{2}=0$. Long dashed line for $\kappa_{2}=1$. Solid line for $\kappa_{2}=2$.

The solution has been plotted for three different values of constant $\kappa_{2}$. The energy density presents a singular behavior only for $\kappa_{2}=0$, while the other two solutions show a non-singular behavior when $t=0$. The solution for $\kappa_{2}=2$ runs quickly to zero. The bulk viscosity is always a negative time function for $\kappa_{2}=1$ and $\kappa_{2}=2$, but the solution for $\kappa_{2}=0$ is valid only for $t>t_{0}$ since $\Pi(t \rightarrow 0)>0$, which means that it lacks of physical meaning in the interval of time $t \in\left(0, t_{0}\right)$. The entropy always behaves like a growing time function but for the case $\kappa_{2}=0$ the universe starts with a non-vanishing entropy, i.e., $\Sigma(0)=$ const., while for the other two solutions $\Sigma(0) \rightarrow 0$. The plots in Fig. [5 show that a large amount of entropy is produced during the expansion of the universe. Regarding the deceleration parameter, the plotted solutions run to an acceleration region since $q(t) \rightarrow-1 / 2$ in a finite time. For this reason, the solution starts in an equilibrium regimen but quickly run to a non-equilibrium state as shown by plots of $l(t)$. A particular solution of this case has been studied by Harko et al [22] obtaining different behavior of the FE main quantities.

\section{C. $\quad$ Case $s=1$.}

The second important case considered corresponds to $s=1$. According to Eqs. (22) and (23), this solution is valid only for the equation of state with $\gamma=\sqrt[3]{2} \approx 1.25992$. Other authors have already studied similar cases for $s=1$, but with different equation of state (see for instance [23] with $\gamma=2$ ) obtaining different results. Then, according to Eqs. (33) and (34), the following particular parametric solution is obtained:

$$
y(\eta)=\left(\kappa_{2} e^{a_{1} D \eta}-\frac{E}{D}\right)^{2}, \quad \text { and } \quad t(\eta)=\frac{1}{E a_{1}}\left[\ln \left(-\frac{E}{D k_{2}}+e^{\eta D a_{1}}\right)-\eta D a_{1}\right] .
$$

Then, the FE main dynamical variables can be explicitly obtained through Eqs. (24)-(31).

In Figs. 7 and 8 , the behavior of the main quantities by giving different values to the constant $\kappa_{2}$ has been plotted.

As we can see the solution is valid only for $t>t_{0}$. The energy density is a decreasing function, but the function behaves like a constant for a $t>t_{c}$. The behavior of the bulk viscous parameter shows that the solution is valid only for $t>t_{0}$ since the solution is positive when $t \rightarrow 0$, decreasing and going to a negative constant value during the cosmological evolution, which is consistent from the thermodynamical point of view. In the same way, the entropy behaves like a growing function only for $t>t_{0}$, showing that a large amount of comoving entropy is produced. Nevertheless, the deceleration parameter shows that the universe starts in a non-inflationary phase, but quickly entering a inflationary one since $q \rightarrow-1 \forall \kappa_{2}$. The plots of $l(t)$ show that plotted solutions are far from equilibrium since they are inflationary solutions. 

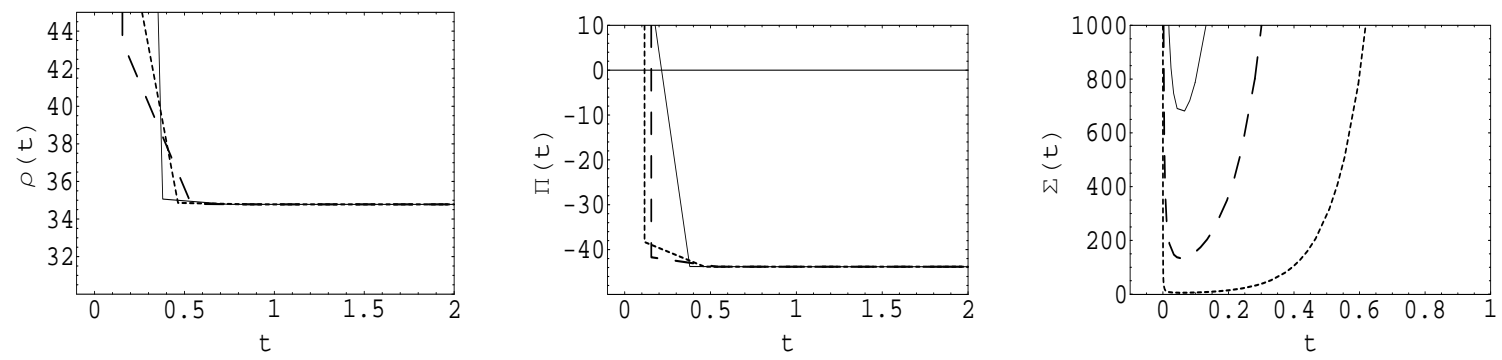

FIG. 7: Solution with $s=1$. Plots of energy density $\rho(t)$, bulk viscosity $\Pi(t)$ and entropy $\Sigma(t)$. Dashed line for $\kappa_{2}=0.1$. Long dashed line for $\kappa_{2}=10$. Solid line for $\kappa_{2}=100$.
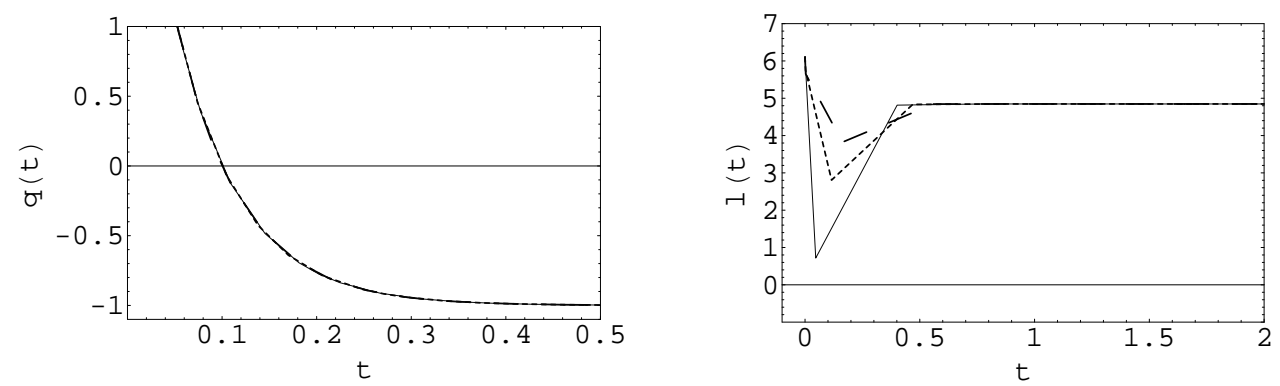

FIG. 8: Solution with $s=1$. Plots of the deceleration parameter $q(t)$ and parameter $l(t)$. Dashed line for $\kappa_{2}=0.1$. Long dashed line for $\kappa_{2}=10$. Solid line for $\kappa_{2}=100$.

\section{SOLUTION WITH $s=1 / 2$.}

We consider now the very special case $s=1 / 2$. This has been the most important and studied case (see for example example [18], [19], 20], 21]) within the framework of the bulk viscous cosmological models since, as it has been pointed out for several authors, this solution is stable from the dynamical systems point of view [26] as well as from the renormalization group approach [27].

In this case, Eq. (91) reduces to:

$$
\ddot{H}-A_{1} \frac{\dot{H}^{2}}{H}+\left(3+C_{1}\right) H \dot{H}+\left(D_{1}+E_{1}\right) H^{3}=0,
$$

where $A_{1}=(1+r)=2-\frac{1}{\gamma}, C_{1}=\sqrt{3}, D_{1}=\frac{9}{4}(\gamma-2), E_{1}=\frac{3}{2} \sqrt{3} \gamma$, and $r=1-1 / \gamma$. Since the coordinate transformation given by Eq. (11) leads to obtain several unphysical solutions for $s=1 / 2$, we perform the more suitable change of variables given as follows (see also [18]),

$$
H=y^{1 / 2}, \quad d \eta=3\left(1+\frac{1}{\sqrt{3}}\right) H d t .
$$

Then, Eq. (43) turns into

$$
y^{\prime \prime}-\frac{A_{1}}{2 y} y^{\prime 2}+y^{\prime}+2 \gamma b y=0,
$$

where $\gamma b=\frac{\sqrt{3}}{8}(\gamma+6)-\frac{3}{2}$. Eq. (45) can be solved by factorization providing new exact parametric solutions for $s=1 / 2$.

Eq. (45) admits the factorization

$$
\left[D-\frac{A}{2 y} y^{\prime}-a_{1}^{-1}\right]\left[D-2 a_{1} \gamma b\right] y=0
$$


which can be rewritten in the form

$$
\begin{aligned}
y^{\prime}-2 a_{1} \gamma b y & =\Omega, \\
\Omega^{\prime}-\left(\frac{A}{2 y} y^{\prime}-a_{1}^{-1}\right) \Omega & =0,
\end{aligned}
$$

or equivalently,

$$
y^{\prime}-2 a_{1} \gamma b y-\mathrm{k}_{1} e^{\eta / a_{1}} y^{A / 2}=0,
$$

where $\mathrm{k}_{1}$ is an integration constant, with solution given as

$$
y(\eta)=e^{2 a_{1} \gamma b \eta}\left(\frac{a_{1} \mathrm{k}_{1} e^{\left(a_{1}^{-1}-a_{1} b\right) \eta}}{2 \gamma\left(1-a_{1}^{2} b\right)}+C_{1}\right)^{2 \gamma}
$$

where $C_{1}$ is an integration constant, and the parameter $a_{1}$ is restricted to values given by

$$
a_{1 \pm}=-\frac{4 \sqrt{3} \gamma \pm \sqrt{\gamma^{2}(72 \sqrt{3}-60)-9 \gamma^{3}+\gamma(432 \sqrt{3}-756)}}{3(\gamma-4 \sqrt{3}+6)}
$$

i.e.,$a_{1+} \in[-64.31,-8.38]$, and $a_{1-} \in[-0.23,-0.01]$. In the following Subsections IV.A and IV.B several possible cases of interest are studied.

\section{A. General solution.}

In this case it is possible to find a explicit parametric equation for $t$ (from Eq. (50) ) with $C_{1} \neq 0$. It is given as follows

$$
t(\eta)=\frac{(\sqrt{3}-3) a_{1}\left(1+\frac{C_{1} \exp \left(\frac{\eta}{2 a_{1 \gamma} \gamma}\left(2 \gamma-a_{1} B\right)\right)}{a_{1} \mathrm{k}_{1}}\right)^{\gamma}}{6 \gamma y^{1 / 2}} F_{1}\left(\frac{-2 \gamma^{2}}{a_{1} B-2 \gamma}, \gamma, 1-\frac{2 \gamma^{2}}{a_{1} B-2 \gamma},-\frac{C_{1} \exp \left(\frac{\eta}{2 a_{1} \gamma}\left(2 \gamma-a_{1} B\right)\right)}{a_{1} \mathrm{k}_{1}}\right) .
$$

To the best of our knowledge the solution given by Eqs. (50) and (52) has not been previously reported.

The FE main dynamical variables are given in parametric form as follows

$$
\begin{aligned}
f(\eta) & =f_{0} \exp \left(\eta-\eta_{0}\right), \\
H(\eta) & =y^{1 / 2}(\eta) \\
q(\eta) & =-\frac{(2+B) C_{1}+2\left(a_{1}+\gamma\right) \mathrm{k}_{1} \exp \left(\frac{\eta}{2 a_{1} \gamma}\left(2 \gamma-a_{1} B\right)\right)}{2\left(C_{1}+a_{1} \mathrm{k}_{1} \exp \left(\frac{\eta}{2 a_{1} \gamma}\left(2 \gamma-a_{1} B\right)\right)\right)} \\
\rho(\eta) & =3 y(\eta), \\
p(\eta) & =3(\gamma-1) y(\eta), \\
\Pi(\eta) & =\frac{C_{1}(B+3 \gamma)+\left(2+3 a_{1}\right) \gamma \mathrm{k}_{1} \exp \left(\frac{\eta}{2 a_{1} \gamma}\left(2 \gamma-a_{1} B\right)\right)}{C_{1}+a_{1} \mathrm{k}_{1} \exp \left(\frac{\eta}{2 a_{1} \gamma}\left(2 \gamma-a_{1} B\right)\right)} y(\eta), \\
\Sigma(\eta) & =\gamma e^{3 \eta}(3 y)^{1 / \gamma} \\
l(\eta) & =\left|\frac{\Pi(\eta)}{p(\eta)}\right|
\end{aligned}
$$

In Figs. 9 and 10, the behavior of the FE main quantities has been plotted. The following constant values have been chosen: $a_{1+}$ as given in Eq. (51) while $B=\frac{\sqrt{3} a_{1}}{4}(\gamma+6)-3 a_{1}, \mathrm{k}_{1}=2, C_{1}=-1$, and $\gamma=1,4 / 3,2$ as usual. The solutions with $a_{1-}$ are unphysical. 

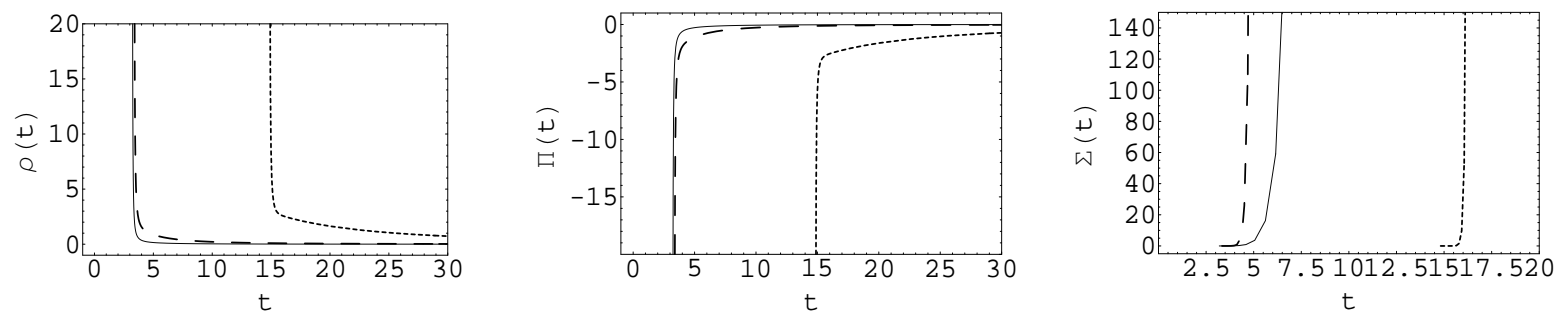

FIG. 9: Solution for $s=1 / 2$. Plots of energy density $\rho(t)$, bulk viscosity $\Pi(t)$ and entropy $\Sigma(t)$. Solid line for $\gamma=2$. Long dashed line for $\gamma=4 / 3$. Dashed line for $\gamma=1$.
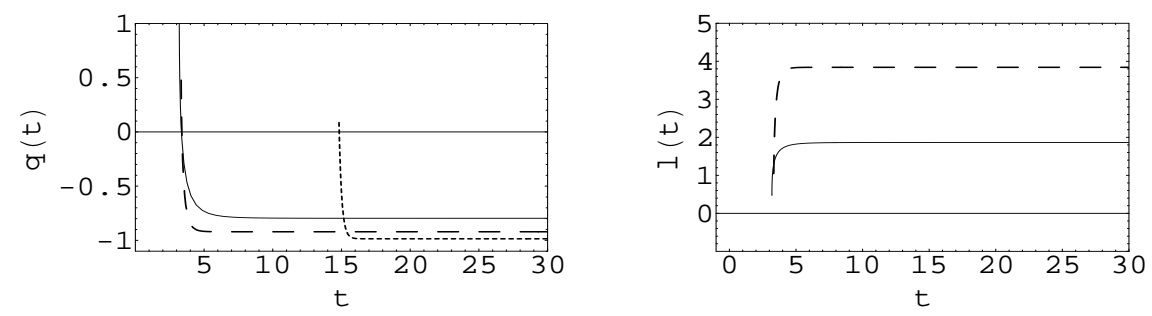

FIG. 10: Solution for $s=1 / 2$. Plots of the deceleration parameter $q(t)$ and parameter $l(t)$. Solid line for $\gamma=2$. Long dashed line for $\gamma=4 / 3$. Dashed line for $\gamma=1$.

The energy density shows a singular behavior as $t \rightarrow 0$, but in a finite time it behaves as a decreasing time function. This solution is valid for all values of time and $\gamma$. The bulk viscous pressure, $\Pi$, is a negative decreasing time function during the cosmological evolution, $\Pi<0 \forall t \in \mathbb{R}^{+}$, as it is expected from a thermodynamical point of view. The viscous pressure also evolves from a singular era but it quickly tends to zero, i.e., in the large limit the viscous pressure vanishes as the viscous coefficient, which also becomes negligible small. The comoving entropy behaves as a growing time function. There exists a fast growth of entropy for $\gamma=4 / 3$, while for $\gamma=1$ the entropy grows slowly. The entropy evolves from a non-singular state, i.e., $\Sigma(0)=0$, but it quickly grows in such a way that a large amount of entropy is produced during the cosmological evolution. The picture of parameter $q(t)$ shows that all the plotted solutions start in a non-inflationary phase, but they quickly run to an inflationary era since this quantity runs to - 1 for all the equations of state. For this reason, the parameter $l(t)$ shows that the solutions are far from equilibrium since they are inflationary solutions.

\section{B. Particular solution}

In the case, it is possible to find a particular solution for $t$ from Eq. (50) with $C_{1}=0$. For this case, the solution simplifies as follows

$$
y(\eta)=\exp (B \eta)\left(\frac{a_{1} \mathrm{k}_{1} \exp \left(\frac{\eta}{2 a_{1} \gamma}\left(2 \gamma-a_{1} B\right)\right)}{2 \gamma-a_{1} B}\right)^{2 \gamma}, \quad \text { and } \quad t(\eta)=(\sqrt{3}-3) \frac{a_{1}}{6 \gamma} y^{-1 / 2} .
$$


Then, the FE main quantities are given in the following form:

$$
\begin{aligned}
f(\eta) & =f_{0} \exp \left(\eta-\eta_{0}\right), \\
H(\eta) & =y^{1 / 2}(\eta), \\
q(\eta) & =-\frac{\left(a_{1}+\gamma\right)}{a_{1}}, \\
\rho(\eta) & =3 y(\eta), \\
p(\eta) & =3(\gamma-1) y(\eta), \\
\Pi(\eta) & =-\frac{\left(2+3 a_{1}\right) \gamma}{a_{1}} y(\eta), \\
l(\eta) & =\frac{1}{3}\left|\frac{\left(2+3 a_{1}\right) \gamma}{a_{1}(\gamma-1)}\right|, \\
\Sigma(\eta) & =\gamma e^{3 \eta}(3 y(\eta))^{1 / \gamma},
\end{aligned}
$$

It is possible to recover the known scaling solution studied by several authors [28], 229] and [30] from Eqs. (62)-(69):

$$
\begin{aligned}
f & =f_{0} t^{H_{0}} \\
H(t) & =H_{0} t^{-1} \\
q(t) & =H_{0}^{-1}-1 \\
\rho(t) & =\rho_{0} t^{-2} \\
p(t) & =3(\gamma-1) \rho_{0} t^{-2}, \Pi(t)=-\Pi_{0} \rho(t), \\
l(t) & =\frac{\Pi_{0}}{3(\gamma-1)}, \\
\Sigma(t) & \approx \frac{\gamma \Sigma_{0}}{3 \gamma H_{0}-2}\left(t^{\frac{1}{\gamma}\left(3 \gamma H_{0}-2\right)}-t_{0}^{\frac{1}{\gamma}\left(3 \gamma H_{0}-2\right)}\right),
\end{aligned}
$$

where $H_{0}=\frac{6 \gamma}{(\sqrt{3}-3) a_{1}}, k_{B}^{-1}=1, \Sigma_{0}=-3 \Pi_{0} H_{0} f_{0}^{3} \rho_{0}^{\frac{1}{\gamma}}\left(\frac{1}{t_{0}}\right)^{3 H_{0}}>0$, and $\Pi_{0}>0$.

In Figs. 11 and 12, the FE main quantities have been plotted using the same numerical values of Figs. 9 and 10.
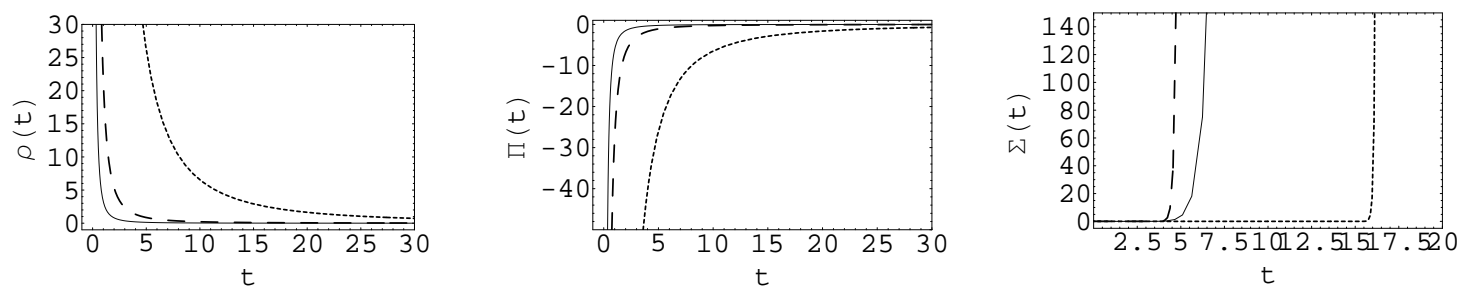

FIG. 11: Solution for $s=1 / 2$ and $C_{1}=0$. Plots of energy density $\rho(t)$, bulk viscosity $\Pi(t)$ and entropy $\Sigma(t)$. Solid line for $\gamma=2$. Long dashed line for $\gamma=4 / 3$. Dashed line for $\gamma=1$.

All the plotted solutions have physical meaning $\forall t$. These solutions and the ones presented in the last solution (with $C_{1} \neq 0$ ) have a similar behavior. We get the following numerical values for parameters $q(t)$ and $l(t): q_{1}=-0.98569$, $q_{4 / 3}=-0.92081, q_{2}=-0.79658$, while $l_{4 / 3}=3.84$ and $l_{2}=1.86$.

\section{SOLUTIONS THROUGH THE LIE GROUP METHOD FOR $s=1 / 2$}

In order to find new solutions and compare with the ones obtained through the factorization method, we study the Hubble rate Eq. (43) with $s=1 / 2$ through Eq. (45) by applying the Lie group method [31]. Eq. (45]) admits the 

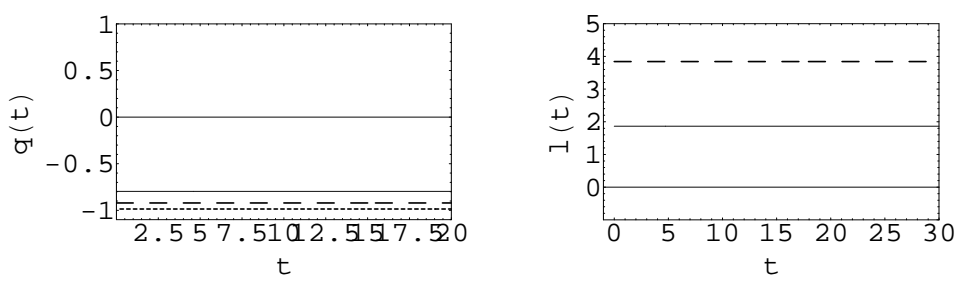

FIG. 12: Solution for $s=1 / 2$ and $C_{1}=0$. Plots of the deceleration parameter $q(t)$ and parameter $l(t)$. Solid line for $\gamma=2$. Long dashed line for $\gamma=4 / 3$. Dashed line for $\gamma=1$.

following symmetries:

$$
\begin{aligned}
\xi_{1} & =[1,0], \quad \xi_{2}=[0, y], \quad \xi_{3}=[1, y] \\
\xi_{4,5} & =\left[0, y^{\frac{A}{2}} \exp \left(\frac{\eta}{2}( \pm a-1)\right)\right], \\
\xi_{6,7} & =\left[y^{\left(1-\frac{A}{2}\right)} \exp \left(\frac{\eta}{2}(1 \mp a)\right), \frac{1 \pm a}{A-2}\left(y^{\left(1-\frac{A}{2}\right)} \exp \left(\frac{\eta}{2}(1 \mp a)\right)\right)\right],
\end{aligned}
$$

where $a=\sqrt{1-8 B+4 A B}$, and $B=\frac{\sqrt{3}}{8}(\gamma+6)-\frac{3}{2}$. The non-zero constants $C_{i j}^{k}$ verifying the relationship $\left[\xi_{i}, \xi_{j}\right]=$ $C_{i j}^{k} \xi_{k}$ are

$$
\left[\xi_{1}, \xi_{4}\right]=C_{14}^{4} \xi_{4}, \quad\left[\xi_{1}, \xi_{5}\right]=C_{15}^{5} \xi_{5}, \quad\left[\xi_{2}, \xi_{4}\right]=C_{24}^{4} \xi_{4}, \quad\left[\xi_{2}, \xi_{5}\right]=C_{25}^{5} \xi_{5}
$$

Then, we shall try to find a suitable change of variables with the symmetries $\xi_{4}$ and $\xi_{5}$. These symmetries, $\xi_{4,5}=$ $\left[0, y^{A / 2} e^{\eta / 2( \pm a-1)}\right]$, bring us to get the following cv that will transform the original ODE into a quadrature. Following the the standard procedure we get:

$$
i=\eta, \quad u(i)=\frac{1}{A-2}\left(e^{\eta / 2(a \mp 1)}\left(y^{1-A / 2}( \pm a-1)+y^{A / 2} y^{\prime}(A-2)\right)\right)
$$

which lead us to obtain the following ODE and the corresponding solution:

$$
u^{\prime}=\mp a u \quad \Longrightarrow \quad u=C_{1} e^{\mp a i} .
$$

Then, the solution to Eq. (45) is given as follows

$$
y_{\mp}=\left(\mp \frac{1}{2 \gamma a} C_{1} e^{\frac{1}{2} \eta(\mp a-1)}+C_{2} e^{\frac{1}{2} \eta( \pm a-1)}\right)^{2 \gamma}
$$

where $a=\sqrt{1+4 B A-8 B}, A=2-\frac{1}{\gamma}$ and $B=\frac{\sqrt{3}}{8}(\gamma+6)-\frac{3}{2}$. In the following Subsections V.A-V.D, the solutions provided in Eq. (81) are separately studied.

\section{A. Solution $y_{-}$with $C_{2} \neq 0$}

For the solution

$$
y_{-}(\eta)=\left(-\frac{1}{2 \gamma a} C_{1} e^{\frac{1}{2} \eta(-a-1)}+C_{2} e^{\frac{1}{2} \eta(a-1)}\right)^{2 \gamma},
$$

with $C_{2} \neq 0$, it is possible to find an explicit parametric equation for $t$ through Eq. (44). It is given as

$$
t_{-}(\eta)=\frac{(3-\sqrt{3})\left(1-\frac{2 a \gamma C_{2} e^{a \eta}}{C_{1}}\right)^{\gamma}}{3(1+a) \gamma y_{-}^{1 / 2}}{ }_{2} F_{1}\left[\gamma, \frac{(1+a) \gamma}{2 a}, \frac{\gamma+a(2+\gamma)}{2 a}, \frac{2 a \gamma C_{2} e^{a \eta}}{C_{1}}\right] .
$$


As we can see, a similar solution to the one obtained through the factorization method has been found. However, as it is shown below, they present several important differences.

The FE main dynamical variables are given in parametric form as follows

$$
\begin{aligned}
f(\eta) & =f_{0} \exp \left(\eta-\eta_{0}\right) \\
H(\eta) & =y_{-}^{1 / 2}(\eta), \\
q(\eta) & =\frac{2 a \gamma C_{2} e^{a \eta}(2+\gamma(a-1))+C_{1}(\gamma(a+1)-2)}{2\left(C_{1}-2 a \gamma C_{2} e^{a \eta}\right)} \\
\rho(\eta) & =3 y_{-}(\eta), \\
p(\eta) & =3(\gamma-1) y_{-}(\eta), \\
\Pi(\eta) & =\frac{\gamma\left(2 a \gamma(a+2) C_{2} e^{a \eta}+C_{1}(a-2)\right)}{C_{1}-2 a \gamma C_{2} e^{a \eta}} y_{-}(\eta), \\
\Sigma(\eta) & =\gamma e^{3 \eta}\left(3 y_{-}(\eta)\right)^{1 / \gamma}, \\
l(\eta)) & =\frac{|\Pi(\eta)|}{p(\eta)},
\end{aligned}
$$

In Figs. 13 and 14 the behavior of the FE main quantities has been plotted.
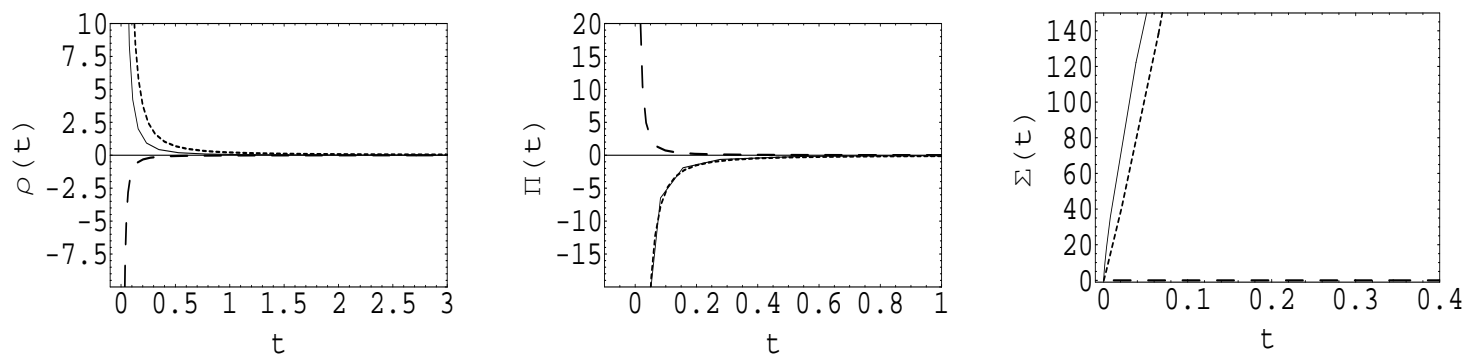

FIG. 13: Solution for $y_{-}$with $C_{2} \neq 0$. Plots of energy density $\rho(t)$, bulk viscosity $\Pi(t)$ and entropy $\Sigma(t)$. Solid line for $\gamma=2$. Long dashed line for $\gamma=4 / 3$. Dashed line for $\gamma=1$.
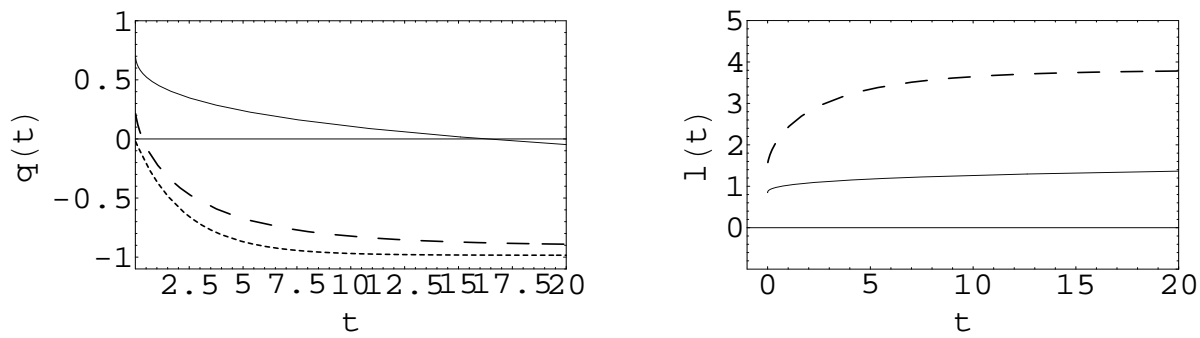

FIG. 14: Solution for $y_{-}$with $C_{2} \neq 0$. Plots of the deceleration parameter $q(t)$ and parameter $l(t)$. Solid line for $\gamma=2$. Long dashed line for $\gamma=4 / 3$. Dashed line for $\gamma=1$.

As it is shown in Fig. 13, the solution is not valid for $\gamma=4 / 3$. For $\gamma=1$ (matter predominance) and $\gamma=2$ (ultra-stiff matter), the energy density behaves as a decreasing time function during the cosmological evolution. This solution is valid for all values of time, except in the case $\gamma=4 / 3$, where $\rho_{4 / 3}<0$. The bulk viscosity is a negative increasing time function, except in the case $\gamma=4 / 3$, where $\Pi_{4 / 3}>0$. The energy-density, bulk viscosity and entropy have a very similar behavior for the cases $\gamma=1$ and $\gamma=2$. The solution has a singular origin since the energy density tends to infinity as $t \rightarrow 0$. The entropy is a growing time function which shows a large amount of comoving entropy during the expansion of the universe. In the case $\gamma=4 / 3$, the entropy starts growing at $t=60$, although we have ruled out this case. The behavior of parameter $q(t)$ shows that the solution for $\gamma=2$ starts in a non-inflationary phase, but after a period of time the solution enters an inflationary era. Nevertheless, the solution for $\gamma=1$ is inflationary 
for all values of $t$. The behavior of parameter $l(t)$ shows that the solution for $\gamma=2$ is close to equilibrium, which is thermodynamically consistent.

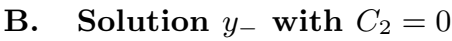

For the case $y_{-}$with $C_{2}=0$, the solution is given by (after simplifying)

$$
y_{-}(\eta)=\left(-\frac{1}{2 \gamma a} C_{1} e^{\frac{1}{2} \eta(-a-1)}\right)^{2 \gamma}, \quad \text { and } \quad t_{-}(\eta)=\int\left(y_{-}\right)^{-1 / 2} d \eta=\frac{(3-\sqrt{3})}{3(1+a) \gamma} y_{-}^{-1 / 2} .
$$

and the FE main dynamical variables are given in parametric form as follows

$$
\begin{aligned}
f(\eta) & =f_{0} \exp \left(\eta-\eta_{0}\right), \\
H(\eta) & =y_{-}^{1 / 2}(\eta), \\
q(\eta) & =\frac{1}{2}((1+a) \gamma-2), \\
\rho(\eta) & =3 y_{-}(\eta), \\
p(\eta) & =3(\gamma-1) y_{-}(\eta), \\
\Pi(\eta) & =(a-2) \gamma y_{-}(\eta), \\
l(\eta) & =\frac{1}{3}\left|\frac{(a-2) \gamma}{(\gamma-1)}\right| \\
\Sigma(\eta) & =\gamma e^{3 \eta}\left(3 y_{-}(\eta)\right)^{1 / \gamma} .
\end{aligned}
$$

The behavior of the FE main quantities has been plotted in Figs. 15 and 16, As it is observed, in this case, we may recover the scaling solution.
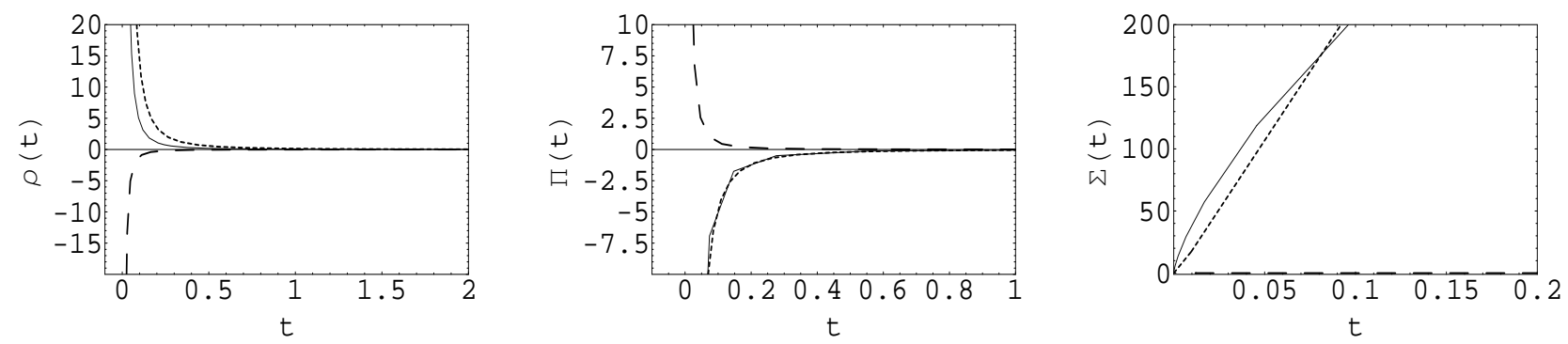

FIG. 15: Solution $y_{-}$with $C_{2}=0$. Plots of energy density $\rho(t)$, bulk viscosity $\Pi(t)$ and entropy $\Sigma(t)$. Solid line for $\gamma=2$. Long dashed line for $\gamma=4 / 3$. Dashed line for $\gamma=1$.

In this case, as in the last solution with $C_{2} \neq 0$, the solution for $\gamma=4 / 3$ is unphysical. All the main quantities behave in the same way as the last solution with $C_{2} \neq 0$ described above. Nevertheless, it is found that $q_{1}=-0.015$ for $\gamma=1$ which represents an inflationary solution, and $q_{2}=0.732$ for $\gamma=2$ which represents a non-inflationary behavior, while $l_{2}=0.845<1$, i.e., the solution is within an equilibrium regime. As it has been shown, most of the known exact solutions of the gravitational $\mathrm{FE}$ with a viscous fluid do not satisfy the condition $l<1$, i.e., the condition of thermodynamic consistency, since they show an inflationary behavior. In the case for $\gamma=2$, we have obtained a solution which is thermodynamically consistent and it may describe the early dynamics of a super-dense post-inflationary era when the dissipative effects produced by the bulk viscosity may play an important role.

\section{Solution $y_{+}$with $C_{2} \neq 0$}

For the solution given by

$$
y_{+}(\eta)=\left(\frac{1}{2 \gamma a} C_{1} e^{\frac{1}{2} \eta(a-1)}+C_{2} e^{-\frac{1}{2} \eta(a+1)}\right)^{2 \gamma},
$$



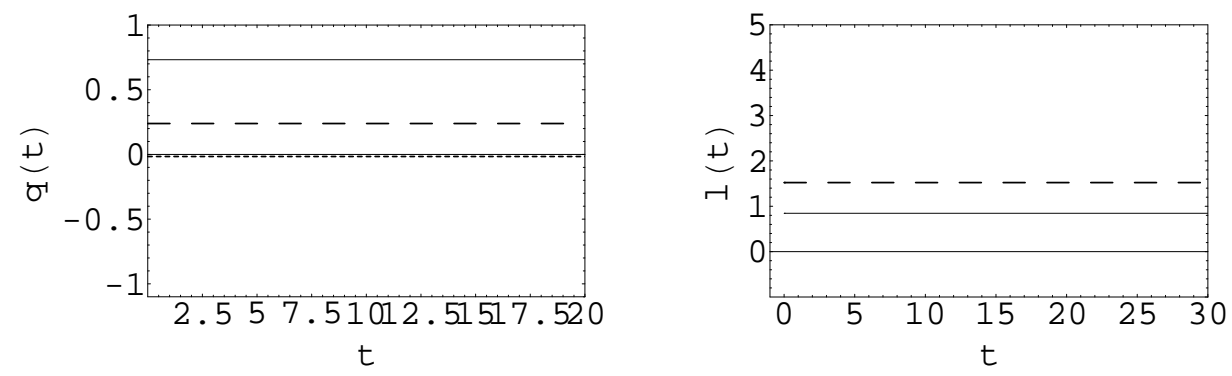

FIG. 16: Solution $y_{-}$with $C_{2}=0$. Plots of the deceleration parameter $q(t)$ and parameter $l(t)$. Solid line for $\gamma=2$. Long dashed line for $\gamma=4 / 3$. Dashed line for $\gamma=1$.

with $C_{2} \neq 0$, we get the explicit parametric equation for the time function

$$
t_{+}(\eta)=\frac{(3-\sqrt{3})}{3} \frac{\left(1+\frac{C_{1} e^{a \eta}}{2 a \gamma C_{2}}\right)^{\gamma}}{(1+a) \gamma y_{-}^{1 / 2}}{ }_{2} F_{1}\left[\gamma, \frac{(1+a) \gamma}{2 a}, \frac{\gamma+a(2+\gamma)}{2 a},-\frac{C_{1} e^{a \eta}}{2 a \gamma C_{2}}\right] .
$$

The main dynamical variables of the FE are given in parametric form as follows

$$
\begin{aligned}
f_{+}(\eta) & =f_{0} \exp \left(\eta-\eta_{0}\right), \\
H_{+}(\eta) & =y_{+}^{1 / 2}(\eta), \\
q_{+}(\eta) & =\frac{2 a \gamma C_{2}(\gamma(a+1)-2)-C_{1} e^{a \eta}(\gamma(a-1)+2)}{2\left(C_{1}-2 a \gamma C_{2} e^{a \eta}\right)}, \\
\rho_{+}(\eta) & =3 y_{+}(\eta), \\
p_{+}(\eta) & =3(\gamma-1) y_{+}(\eta), \\
\Pi_{+}(\eta) & =\frac{\gamma\left(2 a \gamma(a-2) C_{2}-C_{1}(a+2) e^{a \eta}\right)}{C_{1} e^{a \eta}+2 a \gamma C_{2}} y_{+}, \\
l & =\frac{1}{3}\left|\frac{\gamma\left(2 a \gamma(a-2) C_{2}-C_{1}(a+2) e^{a \eta}\right)}{(\gamma-1)\left(C_{1} e^{a \eta}+2 a \gamma C_{2}\right)}\right| \\
\Sigma_{+}(\eta) & =\gamma e^{3 \eta}\left(3 y_{+}\right)^{1 / \gamma}
\end{aligned}
$$

We have plotted the behavior of the FE main quantities in Figs. 17 and 18 .
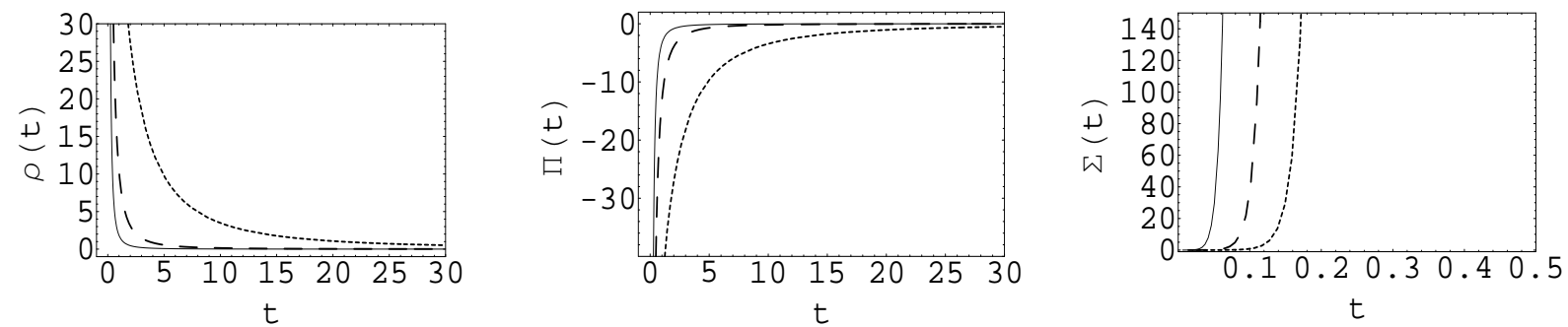

FIG. 17: Solution $y_{+}$with $C_{2} \neq 0$. Plots of energy density $\rho(t)$, bulk viscosity $\Pi(t)$ and entropy $\Sigma(t)$. Solid line for $\gamma=2$. Long dashed line for $\gamma=4 / 3$. Dashed line for $\gamma=1$.

This solution shows a behavior quite similar to the one obtained through the factorization method. The energy density is a decreasing time function and it is valid for all values of time. The bulk viscous pressure is a negative increasing time function, while the entropy is a positive growing time function. As in the case of the factorization method, the obtained solution is valid for all the possible values of parameter $\gamma$. We find a fast growth of entropy for $\gamma=2$, while it grows slowly during the evolution of the universe for $\gamma=1$. The behavior of parameter $q(t)$ shows that all the plotted solutions start in an inflationary phase, since this quantity is close to -1 for every value of $\gamma$. The behavior of parameter $l(t)$ shows that the solutions are far from equilibrium since these are inflationary solutions. 

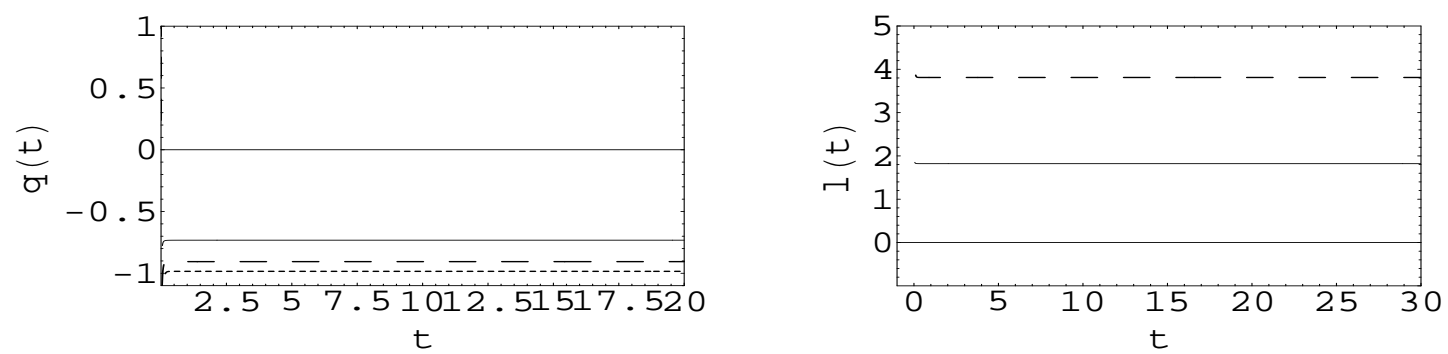

FIG. 18: Solution $y_{+}$with $C_{2} \neq 0$. Plots of the deceleration parameter $q(t)$ and parameter $l(t)$. Solid line for $\gamma=2$. Long dashed line for $\gamma=4 / 3$. Dashed line for $\gamma=1$.

\section{Solution $y_{+}$with $C_{2}=0$}

In the case of solution $y_{+}$with $C_{2}=0$ we get

$$
y_{+}(\eta)=\left(\frac{1}{2 \gamma a} C_{1} e^{\frac{1}{2} \eta(a-1)}\right)^{2 \gamma}, \quad \text { and } \quad t_{+}(\eta)=\frac{(\sqrt{3}-3)}{3(a-1) \gamma} y_{+}^{-1 / 2}
$$

The FE main dynamical variables are given in parametric form as follows:

$$
\begin{aligned}
f(\eta) & =f_{0} \exp \left(\eta-\eta_{0}\right), \\
H(\eta) & =y_{+}^{1 / 2}(\eta), \\
q(\eta) & =\frac{1}{2}((1-a) \gamma-2), \\
\rho(\eta) & =3 y_{+}(\eta), \\
p(\eta) & =3(\gamma-1) y_{+}(\eta), \\
\Pi(\eta) & =(a+2) \gamma y_{+}, \\
l & =\frac{1}{3}\left|\frac{(a+2) \gamma}{(\gamma-1)}\right|, \\
\Sigma(\eta) & =\gamma e^{3 \eta}\left(3 y_{+}\right)^{1 / \gamma} .
\end{aligned}
$$

We may recover the scaling solution as above.
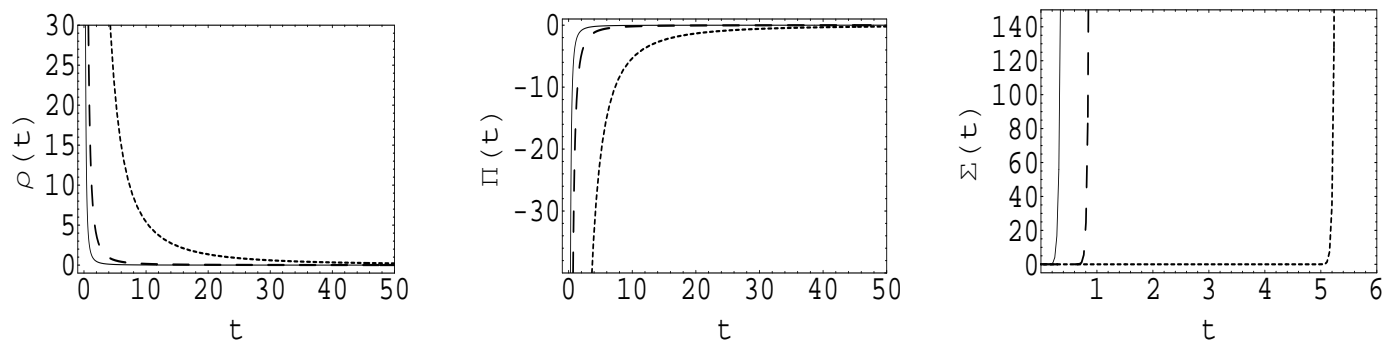

FIG. 19: Solution $y_{+}$with $C_{2}=0$. Plots of energy density $\rho(t)$, bulk viscosity $\Pi(t)$ and entropy $\Sigma(t)$. Solid line for $\gamma=2$. Long dashed line for $\gamma=4 / 3$. Dashed line for $\gamma=1$.

In Figs. 19 and 20 the behavior of the FE main quantities has been plotted. As it can be seen, a very similar behavior to the scaling solution obtained through the factorization method has been obtained. Therefore, we get the same description and conclusions. It is worth mentioning that the following values for the deceleration parameter $q(t)$ are obtained: $q_{1}=-0.984206, q_{4 / 3}=-0.905604$, and $q_{2}=-0.732051$, while for parameter $l(t)$ we obtain $l_{4 / 3}=3.81121$, and $l_{2}=1.82137$, i.e., the same values as the ones obtained for the scaling solution. 

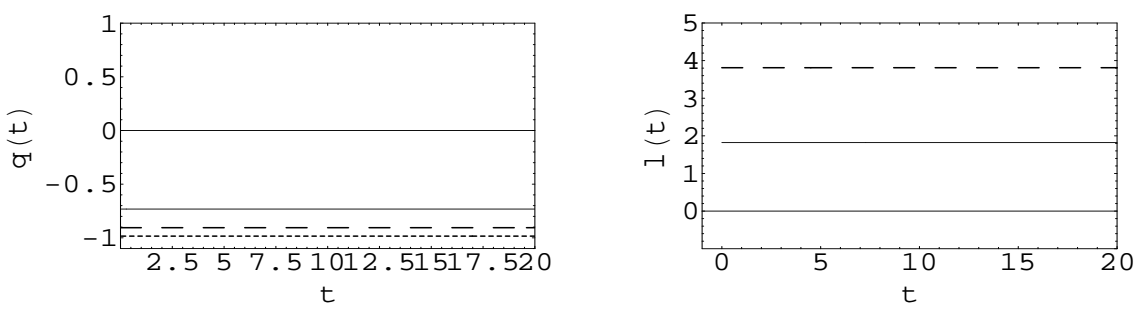

FIG. 20: Solution $y_{+}$with $C_{2}=0$. Plots of the deceleration parameter $q(t)$ and parameter $l(t)$. Solid line for $\gamma=2$. Long dashed line for $\gamma=4 / 3$. Dashed line for $\gamma=1$.

\section{CONCLUSIONS.}

In this work, we have studied a flat FRW cosmological model with a matter model described as a full causal bulk viscous fluid. By assuming the state equations given in Eq. (7), the cosmological model simplifies to a nonlinear second order ODE, the Hubble rate equation, for which a coordinate transformation is performed in order to apply the factorization method. Due to the coordinate transformation developed on the Hubble rate equation, parametric exact solutions have been found. The standard procedure of factorization provides the first order ODE (20), and the restriction condition given in Eq. (21) which provides a relationship between the viscous parameter $s$ and $\gamma$. Then, the analysis developed through factorization allows to study the model for all the values of $s$ determined by Eq. (22), instead of constructing a particular ODE for a single given value of $s$ and arbitrary or specific values of $\gamma$, as it has been previously studied by several authors.

We have studied several models for different values of $s$. Firstly, we have studied and discussed the model for $s=0$, and $\gamma=\sqrt[3]{2}$. The second model is studied for $s=1 / 4$, and $\gamma=2$, finding two solutions. The third case corresponds to $s=1$, and $\gamma=\sqrt[3]{2} \approx 1.25992$. For the very special case $s=1 / 2$, the restriction equation (21) provides the explicit form of parameter $a_{1}$. However, the obtained solutions have not restriction on the values of $\gamma$. For this important case, we have been able to obtain a new solution which reduces, as particular solution, to the known scaling solution. To the best of our knowledge, the parametric solutions obtained for all these cases are new.

In order to obtain more new solutions, the case $s=1 / 2$ has been studied through the Lie group method. The analysis carried out allows to obtain two solutions. The solution (82)-(83) is new, and solution (101)-(102) presents the same behavior as the one obtained through the factorization method. Regarding the solution (82)-(83), it is pointed out that it is not valid for all state equation $\gamma$. It has been shown that for $\gamma=4 / 3$ the solution is unphysical, while for $\gamma=2$ it is thermodynamically consistent and could be relevant from the cosmological point of view.

[1] B. Mielnik, O. Rosas-Ortiz, J. Phys. A.: Math. Gen. 37 (2004) 10007.

[2] H. C. Rosu, Short survey of Darboux transformations, in Symmetries in Quantum Mechanics and Quantum Optics, Eds. F. J. Herranz, A. Ballesteros, L. M. Nieto, J. Negro, C. M. Pereña, Servicio de Publicaciones de la Universidad de Burgos, Burgos, Spain, 1999.

[3] L. M. Berkovich, Sov. Math. Dokl. 45 (1992) 162.

[4] O. Cornejo-Pérez and H. C. Rosu, Prog. Theor. Phys. 114 (2005) 533. H. C. Rosu and O. Cornejo-Pérez, Phys. Rev. E 71 (2005) 046607.

[5] D. S. Wang and H. Li, J. Math. Anal. Appl. 343 (2008) 273.

[6] O. Cornejo-Pérez, J. Phys. A: Math. Theor. 42 (2009) 035204.

[7] P. G. Estévez, S. Kuru, J. Negro, L. M. Nieto, J. Phys. A: Math. Gen. 39 (2006) 3911441. P. G. Estévez, S. Kuru, J. Negro, L. M. Nieto, J. Phys. A: Math. Theor. 40 (2007) 9819.

[8] C. W. Misner, Phys. Rev. Lett. 19, 533 (1966).

[9] L. P. Chimento and A. Jakubi, Phys. Lett. A212, 320 (1996).

[10] C. Eckart, Phys. Rev. 58, 919 (1940).

[11] L. D. Landau and E. M. Lifshitz, Fluid Mechanics, Oxford: Butterworth Heinemann (1987).

[12] W. Israel, Ann. Phys. (NY) 100, 310 (1976).

[13] W. Israel W and J. M. Stewart, Phys. Lett. A 58, 213 (1976).

[14] W. A. Hiscock and L. Lindblom, Ann. Phys. (NY) 151, 466 (1989).

[15] W. A. Hiscock and J. Salmonson, Phys. Rev. D43, 3249 (1991).

[16] W. A. Hiscock and L. Lindblom, Phys. Rev. D35, 3723 (1987). 
[17] R. Maartens, Class. Quantum Grav. 12, 1455 (1995).

[18] L. P. Chimento, A. S. Jakubi. Class. Quantum Grav.10,2047 (1993). Phys. Lett. A 212, 320 (1996).

[19] L. P. Chimento, A. S. Jakubi, V. Mendez and R. Maartens, Class. Quantum Grav. 14, 3363 (1997). L. P. Chimento and A. S. Jakubi, Class. Quantum Grav. 14, 1811 (1997).

[20] M. K. Mak and T. Harko. Gen. Rel. Grav. 30, 1171 (1998). M. K. Mak and T. Harko, Gen. Rel. Grav. 31, 273 (1999).

[21] M. K. Mak and T. Harko. J. Math. Phys. 39, 5458 (1998).

[22] M. K. Mak and T. Harko. Australian Journal of Physics. 53,241 (2000).

[23] M. K. Mak and T. Harko. Euro. Phys. Lett. 56, 762 (2001).

[24] T. Harko and M. K. Mak. IJTP. 38, 1561 (1999).

[25] M. K. Mak and T. Harko, IJMPD 13, 273 (2004).

[26] A. A. Coley, R. J. van den Hoogen and R. Maartens, Phys. Rev. D54, 1393 (1996).

[27] J. A. Belinchón, T. Harko and M. K. Mak. Class. Quantum Grav. 19,3003 (2002).

[28] W. Zimdahl and A. B. Balakin. Entropy 4, 49 (2002).

[29] R. A. Daishev and W. Zimdahl. Class. Quantum Grav. 20, 5017 (2003).

[30] J. A. Belinchón. qr-qc/0412092

[31] N. H. Ibragimov, "Elementary Lie Group Analysis and Ordinary Differential Equations". Jonh Wiley \& Sons, (1999). G. W. Bluman and S. C. Anco "Symmetry and Integral Methods for Differential Equations". Springer-Verlang (2002). 